\title{
Sicher durch den „Analysedschungel“
}

Kontrolle der Praxisbuchhaltung 2017. Das letzte Quartal des laufenden Wirtschaftsjahres ist gut geeignet, um die komplette Jahresbuchhaltung des Jahres 2017 etwas genauer unter die Lupe zu nehmen. Mit diesen zehn Tipps zur Kontrolle der Jahresbuchhaltung kommt man gut durch den Analysedschungel.

AUTOR: BARBARA MERTENS

1. Überprüfen Sie, ob Ihnen alle Begriffe in der Buchhaltung beziehungsweise in der betriebswirtschaftlichen Auswertung (BWA) geläufig sind und Sie ganz genau wissen, was sich dahinter verbirgt.

2. Vergleichen Sie die Summen der einzelnen Erlös- und Kostenkonten mit denen des Vorjahres und versuchen Sie, die Ursachen für Veränderungen zu finden. Dies nennt man Periodenvergleich.

3. Fordern Sie die Sachkontenblätter vom Steuerbüro an, damit Sie bei Bedarf die einzelnen Buchungen anschauen können. Dies macht vor allem dann Sinn, wenn Abweichungen zu Vorperioden nicht erklärlich sind.

4. Rechnen Sie Ihre Rentabilität aus, indem sie den steuerlichen Jahresüberschuss durch die Einnahmen (abzüglich Fremdlabor) dividieren. Die durchschnittliche Rentabilität liegt laut KZBV Jahrbuch bei etwa 39 Prozent, wenn man die Einnahmen um das Fremdlabor bereinigt. Ohne diese Korrektur liegt der Durchschnitt bei 33 Prozent.

5. Kontrollieren Sie, vorausgesetzt Sie sind umsatzsteuerpflichtig, ob der
Vorsteuerabzug so erfolgt, wie es der Realität entspricht. Hier gibt es häufig Kommunikationsprobleme zwischen der Praxis und dem Steuerbüro. Beispielhaft sei die Verbuchung von steuerabzugsfähigen Materialeinkäufen für das Labor genannt, welche auf den Belegen klar gekennzeichnet werden muss.

6. Besprechen Sie mit Ihrem Steuerberater, ob es Sinn macht, zukünftig die allgemeinen Praxiskosten mit der „aufzuteilenden Vorsteuer“ $z u$ buchen. Für umsatzsteuerpflichtige Praxen führt dies zu einer zusätzlichen Vorsteuererstattung.

7. Vergleichen Sie die Summe der gezahlten Einnahmen laut Rechnungsliste in Ihrer Zahnarztsoftware grob mit den geflossenen Einnahmen laut Buchhaltung. Achtung: Hier kann es zu Abweichungen aus der zeitlich versetzten Zahlungsweise zum Jahreswechsel kommen.

8. Machen Sie in der Praxis regelmäßig einen Kassensturz, um zu sehen, ob genau so viel Geld in der Praxiskasse ist, wie laut Kassenbuch darin sein müsste. Dieser Kassensturz muss nach dem GoBD-Leitfaden ohnehin

\section{Der FVDZ unterstützt}

$\triangle$ Mehr Infos unter www.fibu-doc.de. Anwender von fibu-doc erhalten Support über die Hotline.

$\triangle$ Das in den Artikeln dargestellte Buchhaltungskonzept basiert auf der zahnarztspezifischen Buchhaltungssoftware fibu-doc und wird vom FVDZ unterstützt.

regelmäßig erfolgen und dokumentiert werden.

9. Schauen Sie sich die Darlehensbestände in aller Ruhe an. Sind alle Tilgungen richtig verbucht? Dies ersehen Sie aus dem Vergleich von Anfangs- und Endbeständen mit den Kontenauszügen Ihrer Banken.

10. Kontrollieren Sie wenigstens einmal im Jahr, am besten nach der Erstellung des Jahresabschlusses, ob alle dort aufgelisteten Wirtschaftsgüter wirklich noch in der Praxis sind. Wenn nicht, sollte der Anlagespiegel angepasst werden.

Unser Tipp-- Die Anwender von fibu-doc finden eine Vielzahl von Kennzahlen aufbereitet unter dem Button „Kennzahlen“ innerhalb der BWA.

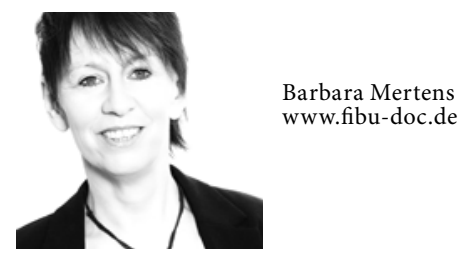

